\title{
Genèse et réception de la théorie de la dégénérescence
}

Contribution à l'analyse des conditions de succès d'une idéologie scientifique

Genesis and reception of the degeneration theory: a contribution to the analysis of the success conditions of a scientific ideology

\section{Patrice Pinell}

\section{OpenEdition \\ Journals}

Édition électronique

URL : http://journals.openedition.org/ress/3507

DOI : $10.4000 /$ ress.3507

ISBN : $1663-4446$

ISSN : 1663-4446

Éditeur

Librairie Droz

\section{Édition imprimée}

Date de publication : 15 mai 2016

Pagination : 183-200

ISSN : 0048-8046

\section{Référence électronique}

Patrice Pinell, «Genèse et réception de la théorie de la dégénérescence », Revue européenne des sciences sociales [En ligne], 54-1 | 2016, mis en ligne le 15 mai 2019, consulté le 21 décembre 2020. URL : http://journals.openedition.org/ress/3507 ; DOI : https://doi.org/10.4000/ress.3507 


\title{
GENÈSE ET RÉCEPTION DE LA THÉORIE DE LA DÉGÉNÉRESCENCE CONTRIBUTION À L'ANALYSE
DES CONDITIONS DE SUCCÈS D'UNE IDÉOLOGIE SCIENTIFIQUE
}

PATRICE PINELL

CNRS, Paris / Université Paris I- CESSP patrice.pinell@cse.cnrs.fr

\begin{abstract}
Résumé. Avec sa théorie de la dégénérescence, l'aliéniste Bénédict Augustin Morel proposait un cadre théorique d'interprétation de la maladie mentale qui allait devenir, pendant trois quart de siècle, l'idéologie scientifique dominant la pensée sur les causes de la folie. Partant de l'hypothèse que le succès rencontré par cette théorie réside dans les solutions conceptuelles et dans les perspectives pratiques qu'elle propose pour résoudre la crise que connaît alors le monde de la psychiatrie, cet article explore deux lignes de recherche. La première s'attache à comprendre en quoi sa théorie renouvelle l'approche de la folie et pourquoi elle est reçue dans le champ médical comme une contribution de premier plan à la psychiatrie. La seconde s'intéresse à Morel en tant qu'agent producteur, pour identifier les propriétés sociales attachées à sa personne, susceptibles de lui conférer une autorité médicale telle que son discours soit reconnu comme une œuvre scientifique majeure.
\end{abstract}

Mots-clés: administration de la preuve, folie, idéologie scientifique, prédisposition héréditaire, théorie organiciste, trajectoire intellectuelle et professionnelle.

\begin{abstract}
The French alienist Bénédict Augustin Morel built a theoretical frame of interpretation of mental illness, the degeneration theory, which became the scientific ideology dominating the understanding of the causes of madness, for several decades. Assuming that the success met by this theory lies in the conceptual solutions and in the practical perspectives it proposes to solve the crisis affecting the French psychiatry at that point in time, this paper investigates two main lines of research. First, it attempts to understand to what extent his theory renews the approach of madness and why it is received in the medical field as a leading contribution to psychiatry. Then, it takes an interest in Morel as producing agent and aims at identifying the social properties attached to his person, liable to confer a medical authority upon him such as his discourse may be recognized as a major scientific work.
\end{abstract}

Keywords : hereditary predisposition, intellectual and professional trajectory, procedures of evidence, organicism, scientific ideology. 
Les débats qui animent, depuis un certain temps déjà, le sous-champ de la psychiatrie, semblent redonner un regain d'actualité à la théorie de la dégénérescence élaborée au milieu du XIX siècle par l'aliéniste Bénédict Augustin Morel. Celle-ci, exposée dans deux ouvrages complémentaires, le Traité des dégénérescences physiques, intellectuelles et morales de l'espèce humaine en 1857 (TD) et dans le Traité des maladies mentales en I860 (TMM), développe une conception résolument organiciste de la folie et accorde un rôle central à la notion de transmission d'une prédisposition héréditaire. Elle inaugure une approche de la causalité qui, refusant l'opposition de l'inné et de l'acquis, fait de la folie la résultante d'un processus au cours duquel, causes physiques, causes morales et prédisposition héréditaire combinent leurs actions. La volonté qui anime Morel de «répondre dans la limite de mes forces, aux aspirations scientifiques de l'époque de progrès où nous vivons» et l'ambition de son projet de «faire avancer la science de l'étude réciproque du physique et du moral» (TD, p.v) annoncent clairement une prétention de faire œuvre de science. Mais, en l'état des savoirs de l'époque sur les mécanismes de l'hérédité, il ne pouvait que bâtir une théorie spéculative, à partir d'un ensemble de présupposés échappant à toute possibilité de vérification, pour produire ce que Georges Canguilhem définit comme une idéologie scientifique (Canguilhem, 198I [1977]). Après avoir occupé plus d'un demi-siècle le devant de la scène médicale, la théorie de la dégénérescence perd son crédit à mesure que la génétique mendélienne s'impose comme science légitime de l'hérédité et relance, au sein de la psychiatrie, la querelle de l'inné et de l'acquis. Et ce, jusqu’à ce que la mise en évidence de l'existence de gènes de prédisposition pour différentes maladies, réactualise la possibilité de penser la causalité dans les pathologies mentales en termes de combinaison entre facteurs héréditaires et facteurs environnementaux. La recherche de gènes prédisposant à l'autisme, à l'hyperactivité, à la déficience intellectuelle (et bien d'autres choses encore), s'accompagne d'une production de discours, importante et variée, allant de la publication scientifique à l'article de vulgarisation. Le débat que suscitent ces questions voit s'exprimer des interrogations légitimes sur le retour au premier plan de discours à fort écho médiatique mettant l'accent sur la prépondérance de la dimension héréditaire dans 
l'étiologie des maladies mentales, quitte à outrepasser les limites du domaine actuel de légitimité de la génétique (Lemerle, 20I4). D’où l'intérêt d'un retour sur l'histoire de cette idéologie scientifique élaborée par Morel au milieu du $\mathrm{XIX}^{\mathrm{e}}$ siècle, non pas tant pour la prendre comme un marqueur d'une époque où la hantise de la dégénérescence de la race se conjugue à la peur des classes «sauvages» (Pick, 1989), mais pour tenter de comprendre les raisons qui ont fait d'elle, pendant trois quart de siècle, la référence en matière d'hérédité pathologique. Dans la mesure où le docteur Morel n'est ni le seul, ni le premier, à traiter des relations entre folie et hérédité, pourquoi sa théorie est-elle, parmi les idéologies scientifiques en concurrence, celle qui réussit à s'imposer dans le champ médical comme la doctrine la plus crédible pour rendre compte de la réalité? Lier son succès au fait qu'elle véhicule un discours réactionnaire - une vision des classes populaires comme foyer de dégénérescence menaçant la société - en phase avec les préoccupations sociales des classes dominantes (Bing, 1994; Darmon, 1989; Dowbiggin, 1985), pose deux types de problèmes. En premier lieu, cette idée suppose que le discours de Morel se distingue de celui de ses contemporains sur le plan idéologique. Or une étude rapide du contenu des thèses avancées par d'autres aliénistes montre qu'il n’en est rien'. Mais surtout, ce type d'explication ne prend pas en compte le fait que ce discours prend son sens et sa valeur symbolique dans un espace spécifique, ici le champ médical, et qu'il doit donc obéir à certaines normes de production propres à ce champ. Car même si la clinique des maladies mentales est

I Pour m'en tenir à l'auteur dont l'ouvrage sur l'hérédité est une source importante d'inspiration pour Morel, à savoir le docteur Prosper Lucas; sa théorie se présente explicitement comme une légitimation de l'ordre social. Distinguant les deux grandes formes que sont l'hérédité de nature et l'hérédité d'institution, il fait de la première, la source de la seconde: «C'est du fait vital que le fait social procède [...] l'hérédité de nature devient en un mot pour nous la raison primordiale et la source réelle de l'hérédité d'institution [...] Dans l'ordre social, (l'hérédité) évoque d'abord la question de principe et de succession de la propriété. Dans l'ordre politique, elle suscite de même la question de principe et de succession de la souveraineté.» (Lucas, |847-|850, p.5). De même, ses diatribes contre le communisme, les longues pages consacrées à la transmission héréditaire de la propension aux excès lubriques, à l'appétit pour l'alcool ou pour la sodomie, ainsi que les chapitres traitant de l'hérédité des propensions au crime contre la propriété et contre les personnes, témoignent d'un souci de donner une légitimité biologique aux normes bourgeoises dominantes qui n'a rien à envier à la théorie de Morel. 
très perméable aux normes morales et comportementales dominantes dans la société, et si l'asile remplit des fonctions de police, un discours psychiatrique doit, pour être reconnu, s'inscrire dans une logique de pensée qui se démarque, au moins sur certains aspects, du sens commun. Tout en intégrant de nombreux présupposés idéologiques, il lui faut mettre en jeu des notions et des concepts ayant cours à l'intérieur du champ médical et par rapport à quoi sa pertinence sera évaluée. Si les deux écrits de Morel se distinguent de la masse des publications «ordinaires », c'est parce quayant posé un diagnostic de crise du savoir et des institutions psychiatriques, ils proposent des éléments de solutions. D’où l'idée que le principal déterminant du succès de la théorie de la dégénérescence réside dans les solutions conceptuelles qu'elle propose pour remédier à cette crise et dans les perspectives pratiques qu'elle ouvre. Cette hypothèse de départ ouvre sur plusieurs lignes de recherche que cet article se propose d'explorer. Il me fallait analyser ces solutions en essayant de dégager en quoi ce qu'elles proposent de nouveau est susceptible de rencontrer une large audience dans l'espace psychiatrique et, au delà, dans l'ensemble du champ. Pour mieux comprendre les propriétés du discours qui donnent à sa théorie une crédibilité scientifique auprès des médecins de l'époque, j’ai essayé de rendre compte de la cohérence entre l'argumentation, le mode d’administration de la preuve et la nature des promesses que la théorie laisse espérer. Mais il me fallait aussi faire porter l'analyse sur Morel en tant qu'agent producteur, de manière à identifier qu'elles étaient les propriétés sociales attachées à sa personne, susceptibles de lui conférer une autorité médicale telle que son discours soit reconnu comme une œuvre scientifique majeure. D’où un travail préalable de reconstitution de sa trajectoire sociale visant à repérer les principales étapes de son parcours intellectuel et professionnel où il acquiert les compétences qu'il mettra en œuvre dans son projet de refonte du savoir psychiatrique. 


\section{LA THÉORIE DE LA DÉGÉNÉRESCENCE COMME PRODUIT D'UNE TRAJECTOIRE ET D'UN ÉTAT DU CHAMP}

Né en I802 dans la Vienne occupée par les armées françaises, Morel ne connaîtra pas sa mère et son militaire de père se débarrassera de lui en le plaçant dans une maison d'éducation religieuse au Luxembourg. Mais il trouve dans l'abbé qui dirige l'institution, ainsi que la servante de ce dernier, les substituts parentaux qui se chargent de l'élever, au point de les suivre lorsqu'ils partent s'installer dans les Vosges. Fortement attaché à sa famille adoptive, il gardera toujours ancrée en lui une croyance dans la doctrine catholique dont l'influence sera déterminante pour la formation de sa pensée, comme pour ses engagements politiques ultérieurs. Cette enfance passée dans des terres de culture germanique lui donne également une parfaite maîtrise de la langue allemande dont il tirera ultérieurement profit (Haupert et al., 2004).

Il a vingt ans quand il part «tenter sa chance» dans la capitale. Un temps tenté par le journalisme, il y renonce pour s'inscrire en I8z I à la faculté de médecine. Sans fortune, obligé de travailler en poursuivant ses études, Morel renonce à préparer l'internat et soutient sa thèse en i839. Durant sa période estudiantine, il aura manifesté une curiosité intellectuelle pour la philosophie et plus encore pour l'histoire naturelle. Il lit Buffon et Cuvier et suit les leçons de Blainville et de Geoffroy Saint-Hilaire. Une fois engagé dans la voie de la médecine aliéniste, il continue de fréquenter le Muséum, se familiarise avec les travaux de Pierre Flourens sur la physiologie du système nerveux, la «génération» ou l'embryologie et y suit les leçons d'anthropologie dispensées par Étienne Augustin Serres. Autant de connaissances acquises qu’il mettra ensuite à profit dans l'élaboration de sa théorie. Sa période parisienne est aussi marquée par des rencontres importantes. En I84I, son ami Claude Bernard, alors interne à l'Hôpital de la Salpêtrière dans le service de Jean-Pierre Falret, le met en contact avec ce dernier, à la recherche d'un traducteur pour aborder la psychiatrie germanique. Cette rencontre avec celui qui deviendra «son maître et son ami» décide de son orientation vers la médecine aliéniste. Elle lui donne en outre l'occasion de travailler sur les écrits des principaux auteurs de «l'école psychique allemande » (Johann Christian August Heinroth, 
Johann Christian Reil, Karl Wilhelm Ideler) qui lui seront une source d'inspiration. Morel, qui a choisi la médecine aliéniste au moment où le dispositif d’asiles d’aliénés départementaux prévu par la loi de I838 se met en place, poursuit sa formation auprès du docteur Ferrus qui est alors l'inspecteur général des services d’aliénés. Ferrus l'envoie à plusieurs reprises en mission visiter les principaux asiles d’aliénés d’Europe. Ces voyages enrichissent sa vision des pratiques de prise en charge et sont autant d'occasions de constituer un réseau de relations scientifiques avec des médecins de plusieurs pays européens.

C'est aussi grâce au docteur Philippe Buchez - un ami proche par ses intérêts pour la psychiatrie et l'histoire naturelle et dont il partage l'engagement dans le catholicisme social - que Morel accède à son premier poste de médecin aliéniste. En I848, Buchez, devenu le président de l’Assemblée nationale, le fait nommer médecin directeur de l’asile d’aliénés de Maréville (Haupert et al., 2004). Cet établissement situé près de Nancy qui reçoit une population de 2000 patients lui fournit son premier terrain d'étude à grande échelle de la folie. Il y prodigue un enseignement clinique ouvert aux médecins et aux étudiants et y entreprend une étude du crétinisme goitreux. Cette pathologie, dont il devient un spécialiste reconnu², sévit à l'état endémique dans la région et c'est avec l'analyse des familles où la maladie touche plusieurs générations, qu'il commence à ébaucher le modèle de causalité au cœur de sa théorie de la dégénérescence. Il décrit un processus pathologique dont la première manifestation est l'apparition d'un goitre, et qui, dans certains cas, va se poursuivre jusqu’à prendre la forme clinique du crétinisme. Si le goitre est la conséquence d'une combinaison de conditions de vie - misère, hygiène déplorable, alimentation insuffisante - avec une constitution géologique du sol produisant des eaux de mauvaise qualité, notamment pauvre en iodure, l'évolution vers le crétinisme met en jeu une cause héréditaire. Car, comme il le constate dans son enquête de terrain, c'est souvent dans des familles de goitreux que naissent des enfants crétins (Morel, I851).

2 Nommé à la commission du goitre et du crétinisme, il élabore en 1864, un programme médico-administratif pour la prise en charge des malades. 
Par ailleurs, Morel est devenu un membre actif de la société médico-psychologique (créée en 1853) qui, à travers ses réunions et la revue qu’elle édite est le principal pôle de production savante et donc de structuration intellectuelle du milieu. Il est donc un aliéniste confirmé, parfaitement familiarisé avec les débats qui agitent la psychiatrie quand, en 1856, il est nommé directeur de l'Asile d’aliénés de Saint-Yon, un établissement qui sous l'impulsion des docteurs Lucien Debouteville, Achille Foville et Jean-Baptiste Parchappe est devenu un haut lieu de la production de statistiques médicales sur la folie (Carbonel, 2005). Il achève alors son livre sur les dégénérescences et élabore son Traité des maladies mentales pour populariser auprès des médecins non aliénistes sa théorie et ses conséquences sur la place de la folie dans le corpus de la pathologie générale.

Le projet de Morel trouve son origine avec les problèmes rencontrés dans sa pratique de médecin asilaire. L'observation clinique d'un grand nombre d'aliénés et les difficultés, voire les échecs fréquents, de leur traitement l'ont convaincu que la voie suivie jusque là dans l'étude de la folie conduisait à une impasse. Lapproche essentiellement descriptive consistant à identifier et à classer les différentes espèces de folie d’après les troubles ou les lésions des facultés intellectuelles et affectives ne permet pas de bien rendre compte des cas rencontrés dans la population des asiles et ne débouche ni sur un traitement rationnel des malades, ni sur des orientations prophylactiques efficaces ${ }^{3}$. L’ambition des deux ouvrages est de renouveler la façon dont, jusque là, la médecine a abordé la caractérisation clinique, le traitement et la prévention des maladies mentales.

Il ne sera pas question avec la théorie de la dégénérescence d’enrichir la caractérisation clinique des hallucinations, des manies, monomanies ou idioties. Ce qui est proposé d'emblée est un changement de perspective sur la folie. Les patients rencontrés dans les asiles, écrit Morel,

3 En cela, ses problèmes font écho à la crise que connaît depuis quelque temps déjà, le milieu des institutions psychiatriques. Crise qui résulte de la conjugaison de plusieurs facteurs: l'échec de plus en plus patent du traitement moral dans lequel, depuis Philippe Pinel, les médecins avaient mis leurs espoirs, l'augmentation constante du nombre des aliénés hospitalisés, l'absence de consensus sur la nature de la folie (maladie organique ou maladie de l'âme) et l'écart qui se creuse entre la psychiatrie et la médecine clinique générale, faute de pouvoir identifier des lésions cérébrales spécifiques (Castel, 1976). 
doivent être considérés comme des représentants de variétés maladives de l'espèce, modifiables dans quelques circonstances, immodifiables dans d'autres [...] ils sont tous plus ou moins frappés du coin de cet état de dégénératif qui les présente à l'observation avec la plupart des caractéristiques propres aux maladies de longue durée et dans lesquelles prédomine la redoutable influence de l'hérédité (TD, p.vi).

C'est la raison pour laquelle l'augmentation constante des aliénés crée pour le pays, une situation morale et physique grave et rend impératif le traitement de la question jusque-là négligée «des causes qui produisent des dégénérescences fixes et permanentes, dont la présence est une menace pour la partie saine de la population » (TD., p.vii).

En mettant au cœur de sa démarche, l’analyse de la folie par l'étude de ses causes et, comme on le verra, des mécanismes par lesquelles elles se combinent pour provoquer une aliénation chronique transmissible à la descendance, Morel se tourne vers la science instituée qu’est la physiologie expérimentale avec laquelle son amitié avec Claude Bernard l’a familiarisé ${ }^{4}$. Mais faire d’une discipline scientifique accordant un rôle central à l'expérimentation animale en laboratoire une source d'inspiration pour l'étude des dégénérescences pose évidemment des problèmes qui ne seront résolus qu'au plan de la rhétorique. Ainsi de l'affirmation de Morel, selon laquelle d'autres voies sont possibles que l'expérimentation sur l'animal pour faire œuvre de science expérimentale dans l'étude des conditions dégénératrices: «il est une foule de circonstances où les solutions que pourrait donner la physiologie expérimentale, sont toutes trouvées par suite de la position déplorables que les causes dégénératrices créent à l'espèce humaine dans des conditions déterminées » (TD., p. xiii). Ce sont les individus vivant dans des milieux pathogènes (logements insalubres, mines, usines), les victimes de l'intoxication alcoolique et de nourritures insuffisantes et/ou altérées qui fournissent au savant «le sujet d'expériences qu’il est inutile de renouveler sur les animaux» (TD, p.xiv). En posant comme allant de soi

4 «Mes rapports non interrompus avec mon ancien ami et condisciple, M. Cl. Bernard, m'ont fait examiner jusqu'à quel point la physiologie expérimentale pouvait éclairer la question des dégénérescence» (TD, p. xiii). 
l'inutilité d'une expérimentation animale qui ne ferait que répéter l'action des causes dégénératrices liées aux conditions d'existence, l'argument occulte deux questions majeures (toujours problématiques aujourd'hui). Celle de la reproduction en laboratoire sur des animaux, des conditions d'existence responsable de la dégénérescence humaine ; et celle de la forme que pourrait prendre l’aliénation chez l'animal. L’idéologie scientifique de la dégénérescence se construit ainsi «dans la méconnaissance des exigences méthodologiques et des possibilités opératoires de la science, dans le secteur de l'expérience qu’elle cherche à investir » (Canguilhem, 1981 [1977], p. 39). Mais cette référence au rôle éclairant de la physiologie expérimentale est importante à prendre en compte par ce qu'elle implique. En effet, elle n'a de sens qu'à deux conditions : donner à la folie un support organique, d'une part, et construire un modèle théorique qui articule l'inné et l'acquis, d’autre part, c'est-à-dire un modèle qui permette de penser les relations que la folie héréditaire entretient avec les conditions physiques, morales et sociales d'existence faisant de l'individu «le sujet d'expériences ». Conditions que Morel remplit en mettant au service d'une vision créationniste des origines de l'homme, des arguments puisés aux sources de l'histoire naturelle et de la physiologie du système nerveux.

Dieu, et ce fut là son chef d'œuvre, a créé le type humain primitif. Mais sa chute du paradis terrestre eut comme conséquence de le placer dans des conditions nouvelles d'existence. L'homme déchu devenu mortel ne pouvait survivre qu'en s'adaptant à son environnement et l'espèce humaine se perpétuer qu'en se reproduisant. Dès lors, elle se trouvait soumise, comme toutes les espèces vivantes, à l'influence de l'hérédité et de toutes les causes susceptibles de produire la maladie, avec des conséquences dont la compréhension, explique Morel, impose un détour par l'histoire naturelle. Il trouve dans les travaux de différents naturalistes les éléments de sa démonstration : l’action combinée de l'hérédité et de conditions d'existence différenciées selon les régions du globe a eu pour résultat une tendance irréversible à faire dévier l'espèce humaine du type primitif (adamique) selon deux modalités : l'une, normale - l'adaptation aux conditions d'existence a produit ces variétés de l'espèce que sont les races -, l'autre, pathologique. C'est à cette dernière que sont dus ces états 
anormaux frappant le physique, l'intelligence et le sens moral qui se retrouvent dans toutes les races comme à toutes les époques et auxquels il donne le nom de dégénérescences. Ces états anormaux se transmettent par l'hérédité parce qu'ils ont un substratum organique siégeant dans le cerveau. En effet, comme Flourens et Franz Joseph Gall l'ont établi, le cerveau est «l'organe de l'âme [...] le siège de toutes les qualités morales et intellectuelles, de la folie comme de la raison [...] dont elle n'est que le trouble» (TMM, p. 7I). Tout en proposant une conception clairement organiciste de la folie, Morel prend position dans la controverse entre les thèses spiritualistes et matérialistes pour renvoyer dos à dos les adversaires. Si, en tant que chrétien, il rejette la vulgate matérialiste faisant du cerveau l'organe producteur de l'intelligence, il ne peut davantage adhérer «aux errements d'un spiritualisme excessif» (ibid.). Car voir dans la folie une maladie de l'âme indépendante de toute atteinte cérébrale, revient in fine à attribuer à l'âme les propriétés d'un corps matériel. Une troisième voie s'impose, déjà ébauchée par le docteur Foville, qui fait du cerveau l'intermédiaire obligé entre l'âme, le reste de l'organisme et le monde extérieur (TMM, p. 74). Dès lors, et tout en respectant le caractère inaltérable de l'âme, le médecin est fondé à concevoir la folie comme un effet des désorganisations de la matière cérébrale s'opposant au libre exercice des facultés intellectuelles, c'est-à-dire comme une maladie qui avec ses caractères spécifiques peut légitimement être rattachée au grand cadre de la pathologie générale. Ainsi Morel rend compatible la doctrine de l'Église dans ce qu'elle a de plus orthodoxe avec une conception organiciste de la folie qui justifie le rattachement de la clinique des maladies mentales à la médecine générale. Un tel discours, parce qu'il peut prétendre à une double légitimité, dans le champ religieux et dans le champ médical, est susceptible de recueillir une large audience. Adossé à cette double légitimité, il se trouve en position favorable pour tenter ce coup de force symbolique qu'est la redéfinition de la clinique de la folie à partir de l'analyse de ses causes. Et cela, en revendiquant une parenté entre son projet théorique et l'ambition de ceux qui, comme Claude Bernard, entendent également renouveler la pensée médicale en liant la clinique à la physiologie expérimentale. Mais, pour faire accepter des vues aussi nouvelles dans un champ dominé par l'élite 
hospitalière parisienne, il lui faut compenser le peu d'autorité scientifique attachée à sa position de médecin d'un asile de province 5 . Aussi ses deux ouvrages vont-ils se présenter comme une somme embrassant un vaste domaine de connaissances pour puiser des arguments bien au delà de la seule clinique des maladies mentales, dans l'histoire naturelle, la physiologie, l'anthropologie, la philosophie, la statistique morale, l'anatomie pathologique. C'est l'ensemble des sciences traitant de l'humain qui seront ainsi convoquées pour installer sa théorie de la folie au centre de leur zone de recoupement.

\section{METTRE DE L'ORDRE DANS LE FOUILLIS DES CAUSES}

La problématique «morélienne» de la folie fait de la nature de la cause sa pierre angulaire. C’est elle qui détermine l'altération des fonctions, et de là, le mode d'évolution de la maladie, son début, sa marche, ses caractères et sa terminaison. Définir et classer les aliénations par la nature de leurs causes est donc la condition pour constituer des catégories «réelles » de malades en faisant émerger ce qu'ils ont en commun. Mais la démarche se heurte à un problème de taille : le grand nombre des causes de tous ordres qui ont été identifiées pourrait condamner à l'inutilité tout effort de classification. Morel le résout en construisant sa problématique de la causalité sur l'articulation de deux idées-clés. L'une fait de la folie chronique le résultat d'un processus pathologique individuel et familial au cours duquel plusieurs types de causes se combinent, tandis que l'autre hiérarchise le rôle des causes dans ce processus selon leur nature.

5 La loi de 1838 en instituant un dispositif national d'asiles d'aliénés départementaux a introduit une division au sein des structures institutionnelles prenant en charge les fous. Jusque là, la prise en charge des fous avait lieu dans des quartiers spéciaux mis en place au sein des hôpitaux publics (à Paris dans les hospices de Bicêtre et de La Salpêtrière) et dirigés par des médecins appartenant à l'élite clinique des médecins des hôpitaux. Si les hôpitaux publics sont gérés par des conseils d'administration au niveau des municipalités, les asiles d'aliénés nouvellement mis en place, parce qu'ils doivent assurer des fonctions de police découlant de la législation sur l'internement d'office, sont placés sous la responsabilité directe des préfets. Pour assurer la direction de ces établissements de soins et d'enfermement, la loi prévoit la création d'un corps de médecins d'asile fonctionnaires. Choisis par le préfet, ces médecins qui ne sont pas passés par la voie d'excellence des concours hospitaliers, jouissent d'une faible considération auprès de l'élite hospitalo-universitaire (voir Pinell, 2005). 
Lapproche en terme de processus renouvelle la question cruciale pour la clinique, de la distinction entre les folies transitoires, les folies chroniques, les folies acquises et celles qui sont héréditaires. Il ne s'agit pas de les considérer comme des catégories ontologiquement différentes, mais de reconstituer les enchaînements de phénomènes pathologiques par lesquels un trouble mental acquis et transitoire peut se chroniciser puis et en venir à se transmettre par les voies de l'hérédité. Si les formes de folies transitoires n’évoluent pas fatalement en folies héréditaires, toutes peuvent, dans certaines circonstances, connaître une telle évolution. Cette problématique guide une analyse visant à mettre de l'ordre dans le fouillis des causes en les différenciant selon leur nature et la façon dont elles agissent. Première distinction, celle faite entre les causes prédisposantes générales et les causes prédisposantes individuelles. Les premières renvoient à tout ce qui, dans une société - la civilisation, la religion, la politique, les mœurs, etc. - est susceptible de favoriser l'apparition de troubles mentaux (d'où la convocation de la philosophie et de l'anthropologie pour en rendre compte). Elles ne sont pas directement responsables de la folie, mais elles constituent un cadre contextuel favorisant l'action des multiples causes prédisposantes individuelles. Ces dernières peuvent être de nature physique (n'importe quelle maladie ou intoxication) ou morales (tous les chagrins, les vices, toutes les formes d'excitation des facultés intellectuelles) ou réalisent une combinaison des deux (l'alcoolisme joue sur les deux registres comme intoxication et comme vice). Si toutes ces causes sont à même de produire une perturbation de l'état mental d'un individu, elles n'en doivent pas moins être différenciées en deux catégories. La première est celle des causes accessoires dont l'action, étant occasionnelle, n’entraîne ni lésions cérébrales ni troubles fonctionnels chroniques ${ }^{6}$. On trouve parmi elles toutes les maladies «non nerveuses » susceptibles, comme par exemple la fièvre typhoïde, de provoquer un délire passager, tous les états liés à la physiologie féminine ${ }^{7}$ et

6 II peut toutefois arriver que la cause occasionnelle s'installe dans la durée (un état pathologique qui se chronicise, un comportement vicieux qui se répète, un chagrin inconsolable). Elle peut alors susciter un trouble durable dans l'organisme, déterminer une lésion cérébrale et produire une aliénation permanente également susceptible d'être transmise aux descendants.

7 Dans la partie consacrée à l'influence de ces états (menstruations, grossesse, etc.) sur le psychisme féminin, où il cite de nombreux médecins, Morel ne fait qu'emprunter aux lieux 
le vaste ensemble des causes morales. La seconde catégorie regroupe les causes spécifiques. Toutes sont susceptibles dans certaines circonstances d'entraîner des troubles invariables et permanents des fonctions du système nerveux responsable d'une aliénation. C'est le cas de certaines névroses (l'hystérie, l'épilepsie et l'hypochondrie), des différentes intoxications (alcool, substances narcotiques, plomb, environnements physiques malsains, etc.), des affections propres du cerveau (congestions, hémorragies, méningites) et de l'hérédité. Les aliénations résultant des lésions cérébrales provoquées par chacune de ces différentes causes spécifiques, possèdent des traits communs qui définissent un type propre à la cause : une folie alcoolique ${ }^{8}$, une folie hystérique ou une folie héréditaire, etc.

\section{LA PRÉDISPOSITION HÉRÉDITAIRE COMME PRINCIPE ORGANISATEUR}

Dans ce tableau des causes où elle est rangée dans la catégorie des causes spécifiques, l'hérédité occupe une place à part du fait de la pluralité de ses modes d'action. L'analyse du rôle que Morel lui fait jouer dans l'enchaînement des causes responsables d'une aliénation, puise à plusieurs sources. Celle, incontournable, du traité publié quelques années plus tôt par Prosper Lucas, le premier a proposer sur l'hérédité en général et sur ses rapports avec les maladies du système nerveux, un véritable état de la question (Lucas, I847-1850). Morel y fait de nombreux emprunts, mais s'écarte d’une conception restée fidèle à la logique descriptive de la classification de Jean-Étienne Esquirol qui s'en tient à montrer, pour chacune des espèces de folie (hallucinations, manie, etc.), l'existence de nombreux cas de transmission héréditaire. Il est surtout redevable à Jacques-Joseph Moreau de Tours de l'idée que l'élément pathologique qui se transmet dans la folie héréditaire est une prédisposition aux maladies du système nerveux. Aussi, la survenue d'une aliénation semblable à celle du

communs des idéologies scientifiques traitant de la nature féminine.

8 «On verra que la répétition fréquente de la même habitude, l'ingestion des boissons alcooliques par exemple, suscite chez les individus des perturbations identiques du système nerveux. C'est le délire avec des hallucinations spéciales; ce sont les mêmes troubles digestifs, les tremblements des membres...» (TMM, p. 262). 
parent n'est qu'un des cas de figures possibles. Le vice héréditaire transmis peut rester sans effet ou se manifester par une altération à peine repérable des facultés intellectuelles ou au contraire plus grave (Moreau de Tours, I850).

Morel reprend la notion de prédisposition pour la mettre au centre de sa théorie, en en faisant le principe organisateur du processus d'enchainement des causes spécifiques et accessoires conduisant à la folie chronique, au sein d’une famille de dégénérés. Le modèle de causalité qu'il élabore se découpe en étapes successives. Tout commence avec un individu soumis à une des causes spécifiques susceptibles de produire des atteintes cérébrales entraînant une folie chronique. Si rien ne vient arrêter le processus pathologique en marche, celui-ci, une fois la folie constituée, peut se poursuivre par la transmission à ses enfants d'une prédisposition héréditaire. C’est le cas si, au moment de la procréation, la cause spécifique continue d'exercer son action sur le géniteur malade. Dans la génération suivante où les dispositions à la folie sont innées, la sensibilité aux causes est plus forte: cette fois, les causes accessoires suffisent à provoquer une folie chronique. Au fil des générations successives, l'effet de la prédisposition héréditaire s’aggrave et la dégénérescence en vient à se manifester dès la naissance par des anomalies physiques congénitales et des troubles plus ou moins massifs de l'intelligence, donnant le jour à des enfants qui, stériles ou morts précocement, seront incapables de perpétuer la lignée. Le rôle central qu'il fait jouer à la prédisposition dans son modèle est aussi ce qui lui permet de rendre compte de la diversité des situations observées. Le fait qu’elle ne manifeste ses effets qu'en combinaison avec l'action de causes prédisposantes explique que les enfants d'une même fratrie issue d’un parent aliéné ne vont pas tous être touchés par la folie et que, dans certains cas, l’aliénation «saute» une génération. En outre, le processus conduisant à la dégénérescence peut être réversible lorsque des circonstances favorables viennent agir dans le sens d’une régénération, si le médecin sait en tirer partie. Un traitement adapté, une éducation hygiénique et morale intelligente, un mariage qui apporte dans la famille un sang nouveau de bonne nature, peuvent corriger la marche de 
l'hérédité en produisant une rénovation heureuse du principe héréditaire, à condition, ici encore, d'être guidé par l'hygiène (TMM, p. 513-517)9

Pour donner un crédit scientifique à ses propos, Morel use d'un mode d’administration de la preuve qui fait, à l'époque, l'objet d'un consensus dans le champ médical. Il va chercher dans les observations cliniques, les faits validant sa théorie. Par exemple, dans la partie où il traite de l'influence des états propres à la physiologie de la femme, il présente une série de cas de délires survenus sous l'action de ces causes accessoires. Si la plupart d'entre eux illustre le caractère passager de l'épisode délirant, il en est quelques-uns où le désordre mental s'installe dans la durée et évolue en folie chronique. Il se trouve que dans chacun de ces cas, des antécédents de folie dans la famille sont retrouvés, laissant penser que la femme était porteuse d’une prédisposition héréditaire. Cette preuve par les faits confirme bien l'idée que des causes accessoires peuvent déterminer la constitution d'une folie chronique lorsqu'elles se combinent à une prédisposition. Toutefois, la force de conviction attachée à ce type d'argumentation alors commun en médecine, tend à varier selon deux paramètres : le nombre des cas évoqués et l’autorité médicale de leurs auteurs. Fort des compétences qu'il a accumulées, Morel est en condition de ne lésiner sur aucun des deux. Il accumule les éléments de preuve en multipliant les exemples cliniques, entrelaçant ceux tirés de sa propre expérience et ceux, nombreux, qu’il emprunte à ses confrères français et étrangers, impliquant dans la construction de son édifice théorique des «faits » établis par tout ce que le champ médical compte comme figures reconnues de la clinique des maladies mentales.

9 Morel s'attache à trouver un traitement adapté à la phase de la maladie (période initiale, rémission) à l'état de l'aliéné (agitation, torpeur, apathie) et aux différentes formes de folie. Sa panoplie thérapeutique qui combine l'emploi des médications classiques avec celle des nouvelles substances issues des progrès de la recherche pharmaceutique (chloroforme, alcaloïdes, bromures), apparaît beaucoup plus riche et diversifiée que celle de ses prédécesseurs. (Ledermann, 1982). 


\section{UN RENOUVELLEMENT DE LA PENSÉE SUR LA FOLIE ET SUR L'HÉRÉDITÉ MORBIDE}

La théorie de la dégénérescence est l'objet d’un rapport élogieux de Buchez à la Société médico-psychologique et vaut aussi à son auteur un prix de l’Académie des sciences. Et les mentions qui en sont faites dans la littérature médicale sont à l'avenant ${ }^{10}$. Il est également l'un des cinq spécialistes des maladies mentales conviés à collaborer au prestigieux Dictionnaire encyclopédique des sciences médicales dirigé par Amédée Dechambre. L’œuvre de Morel convainc parce qu’elle reconnaît sa dette aux productions médicales et scientifiques de son époque, tout en proposant, par un changement de perspectives sur la folie, de dépasser les oppositions canoniques (matérialisme/spiritualisme, acquis/inné) dans lesquelles le débat s'enlisait. Entendant dépasser les approches descriptives pour faire entrer l'étude de la folie dans une médecine de la causalité, Morel est le premier à élaborer une interprétation organiciste dynamique de la folie qui explique le passage du transitoire au chronique et à l'héréditaire. Constituée au niveau individuel et familial, la folie ne se résume pas à l'inscription dans le cerveau d'un individu de désordres physiques, moraux et sociaux et la théorie permet de penser la permanence de l'influence perturbatrice des conditions d'existence sur la succession des générations. Ce recentrage du regard médical, du patient aliéné sur la famille touchée par la dégénérescence, est également riche d’implications sociales. Car si la dégénérescence frappe dans tous les milieux sociaux, elle est plus particulièrement à l'œuvre dans la population ouvrière, où les conditions d'existence des individus sont propices à l'engendrement réciproque des causes néfastes (pauvreté $\leftrightarrow$ alcoolisme $\leftrightarrow$ promiscuité $\leftrightarrow$ instincts vicieux, etc.). Ce qui justifie et l'asile comme lieu permettant d'extraire le malade d'un milieu pathogène, et la nécessité d’interventions préventives ciblant principalement les enfants et axées sur «la moralisation des masses » par la diffusion des règles d'hygiène, le développement de l'instruction et de l'éducation morale. Soit l'action combinée

10 Ainsi Louis Delasiauve, médecin des aliénés à Bicêtre, académicien, éditeur du Journal de Médecine mentale, cite régulièrement les travaux de Morel et le proposera comme membre correspondant de l'Académie de médecine. Benjamin Ball, le premier titulaire de la chaire de clinique des maladies mentales, parle lui, à propos de la dégénérescence, «des pages immortelles» écrites par Morel. 
du corps médical, mais aussi des gouvernements et des administrations locales (TD, p. 680-693). La théorie est donc en phase avec les mouvements soucieux de la régénération de la race française dont l'influence ira grandissante après le désastre de la guerre avec la Prusse. Après la mort de son auteur en I873, la théorie de la dégénérescence commencera une nouvelle carrière. Détaché de ses prémisses créationnistes et inséré, sans perdre sa cohérence, dans une perspective évolutionniste, le mécanisme d’action de l'hérédité imaginé par Morel fournit un cadre conceptuel à la lutte antialcoolique (Dargelos, 2008) ou au courant positiviste en criminologie (Darmon, 1989), en même temps qu'il devient, dans les écrits des élèves de Charcot, la référence théorique dominante en matière d'hérédité morbide (Pinell, 200I). Mais il n’appartient pas à cet article de traiter la question de la longévité de cette idéologie scientifique au delà du contexte qui présidait à sa (bonne) réception initiale.

\section{BIBLIOGRAPHIE}

BING F., 1994, «La théorie de la dégénérescence», in Nouvelle histoire de la psychiatrie, Jacques Postel et Claude Quétel (dir.), Paris, Dunod, p.233-238.

CANGUILHEM G., 198I (1977), Idéologie et rationalité dans l'histoire des sciences de la vie, Paris, Vrin.

CARBONEL F., 2005, «L'asile pour aliénés de Rouen, un laboratoire de statistiques morales de la restauration à $\mid 848 \gg$, Histoire et Mesure, XX-I/2, p.97-136.

CASTEL R., 1976, L’Ordre psychiatrique, Paris, Éditions de Minuit.

DARGELOS B., 2008, La Lutte antialcoolique en France depuis de XIxe siècle, Paris, Dalloz. DARMON P., 1989, Médecins et assassins à la Belle Epoque, Paris, Éditions du Seuil.

DOWBIGGIN I., 1985, «Degeneration and Hereditarianism in French Mental Medicine. 1840-1890: Psychiatric Theory as Ideological Adaptation », in The Anatomy of Madness, t. I, W. F. Bynum, R. Porter (dir.), London, Tavistock, p. 188-232.

HAUPERT J., DE SMET Y. et SPAUTZ J.-M., 2004, « La Théorie de la dégénérescence de Bénédict Augustin Morel (|809-1873): inspirateurs et thuriféraires》, L'Information psychiatrique, 80-I, p.43-49. 
LEDERMANN F., 1982, «La psychiatrie française et les médicaments: Pomme, Pinel, Esquirol, Morel», Revue d'histoire de la pharmacie, 70-254, p. I89-206.

LEMERLE S., 2014, Le Singe, le Gène et le Neurone. Du retour du biologisme en France, Paris, PUF.

LUCAS P., 1847-1850, Traité philosophique et physiologique de l'hérédité naturelle dans les états de santé et de maladie du système nerveux, t. I, Paris J-B Baillière.

MOREAU DE TOURS J.-J., I850, Un Chapitre oublié de la pathologie mentale, Paris, Masson.

MOREL B.-A, I851, Considérations sur les causes du crétinisme endémique à Rosièresaux-Salines (Meurthe), Nancy, Imp. De Vagner.

-, 1857, Traité des dégénérescences physiques, intellectuelles et morales de l'espèce humaine, Paris, Baillière.

-, 1860, Traité des maladies mentales, Paris, Masson.

PICK D., 1989, Faces of degeneration, Cambridge, Cambridge University Press.

PINELL P., 200I, «Degeneration Theory and Heredity Patterns Between 1850 and 1900», in Heredity and infection. The history of disease transmission, J.-P. Gaudillière et I. Löwy (dir.), London and New York, Routledge, p. 245-260.

-, 2005, «Champ médical et processus de spécialisation», Actes de la recherche en sciences sociales, 156-157, p.4-36. 